\title{
PSEUDO-PONTRJAGIN CLASSES
}

\author{
YASUO MATSUSHITA
}

\begin{abstract}
For a pseudo-Riemannian manifold we can construct a pseudoPontrjagin class as represented by a certain $\operatorname{ad}\left(\mathrm{SO}_{0}(p, q)\right)$-invariant form on the manifold so that it coincides with the Pontrjagin class of the manifold.
\end{abstract}

Let $M$ be an $n$-dimensional, orientable, $C^{\infty}$-differentiable, pseudo-Riemannian manifold with a metric $\langle$,$\rangle of signature$

$$
(+\underset{p}{\cdots}+-\underset{q}{\cdots}-), \quad p \geq q, p+q=n .
$$

By orientability we mean that the structure group of the tangent bundle $\xi$ of $M$ is the identity component $\mathrm{SO}_{0}(p, q)$ of the pseudo-orthogonal group $\mathrm{O}(p, q)$. As an analogue of the Pontrjagin class for a Riemannian manifold, we can define for $M$ a pseudo-Pontrjagin class $\bar{p}$ as an element of the de Rham cohomology ring $H^{*}(M ; \mathbf{R})$ which is represented by a certain ad $\left(\mathrm{SO}_{0}(p, q)\right)$-invariant curvature polynomial. We show that such a class $\overline{\mathfrak{p}}$ coincides with the Pontrjagin class $\mathfrak{p}$ constructed on the intrinsic Riemannian structure of $M$. Such a coincidence has been reported only for the case of the 4-manifolds of metric signature $(++--)$ [2, Proposition 2]. This paper heavily depends on Chern's analysis [1] of the generalized Gauss-Bonnet formula for the Euler characteristics of pseudo-Riemannian manifolds.

At each point of $M$ we can choose a local pseudo-orthonormal basis $\left\{e^{i}\right\}(i=$ $1, \ldots, n)$ of 1 -forms so that $\left\langle e^{i}, e^{j}\right\rangle=\varepsilon_{i} \delta_{i j}\left(\delta_{i j}\right.$ : Kronecker delta), where $\varepsilon_{i}=1$ for $1 \leq i \leq p$ and $\varepsilon_{i}=-1$ for $p+1 \leq i \leq n$. The pseudo-Riemannian curvature tensor $R=\left(R^{i j}{ }_{k m}\right)$ (of type (2.2)) defines a linear transformation on the bundle $\Lambda^{2}$ of 2-forms over $M$ :

$$
R\left(e^{i} \wedge e^{j}\right) \equiv \Omega^{i j}=\frac{1}{2} \sum_{k, m} R^{i j}{ }_{k m} e^{k} \wedge e^{m},
$$

where $\Omega^{i j}$ are the components of the curvature 2 -form with values in the Lie algebra $\mathfrak{s o}(p, q)$.

The $n \times n$ matrix $\Omega=\left[\Omega^{i j}\right]$ is antisymmetric: $\Omega+{ }^{t} \Omega=0$. The curvature 2 -form $\Omega^{\prime} \equiv\left[\Omega_{i}{ }^{j}\right]$ of type (1.1) is related to $\Omega$ as follows:

$$
\Omega=I_{p, q} \Omega^{\prime}, \quad I_{p, q}=\left[\begin{array}{cc}
I_{p} & 0 \\
0 & -I_{q}
\end{array}\right],
$$

where $I_{k}=\operatorname{diag}[+1, \ldots,+1](+1: k$ times $)$. Thus, $\Omega^{\prime}$ satisfies $I_{p, q} \Omega^{\prime}+{ }^{\mathrm{t}} \Omega^{\prime} I_{p, q}=$ 0 . Under the actions of the group $\mathrm{SO}_{0}(p, q)$, the curvature is transformed in the

Received by the editors May 29, 1984 .

1980 Mathematics Subject Classification. Primary 53C50, 57R.20.

Key words and phrases. Pseudo-Riemannian manifolds, pseudo-Pontrjagin classes, Pontrjagin classes. 
following way:

$$
\begin{gathered}
\Omega^{\prime} \mapsto \bar{\Omega}^{\prime}=g^{-1} \Omega^{\prime} g, \\
\Omega \mapsto \bar{\Omega}={ }^{\mathrm{t}} g \Omega g,
\end{gathered}
$$

where $g \in \mathrm{SO}_{0}(p, q)$.

Let $\mathfrak{M}_{n}(\mathbf{R})$ denote the algebra of all real $n \times n$ matrices. We now define a function $f: \mathbf{R} \times \mathfrak{M}_{n}(\mathbf{R}) \rightarrow \mathbf{R}$ by

$$
f(t, X)=(-1)^{q} \operatorname{det}\left(t I_{p, q}-(2 \pi)^{-1} X\right) .
$$

For this function we have

LEMMA. $f(t, \Omega)$ is invariant under the actions of $\mathrm{SO}_{0}(p, q)$.

ProOF. First we must note that every element $g \in \mathrm{SO}_{0}(p, q)$ satisfies ${ }^{\mathrm{t}} g I_{p, q} g=$ $I_{p, q}$. From the transformation rule (2) for $\Omega$, together with the above relation, we have

$$
\begin{aligned}
f(t, \bar{\Omega}) & =(-1)^{q} \operatorname{det}\left(t I_{p, q}-(2 \pi)^{-1} \bar{\Omega}\right) \\
& =(-1)^{q} \operatorname{det}\left(t I_{p, q}-(2 \pi)^{-1} \mathrm{t} g \Omega g\right) \\
& =(-1)^{q} \operatorname{det}\left(t^{\mathrm{t}} g I_{p, q} g-(2 \pi)^{-1} \mathrm{t} g \Omega g\right) \\
& =(-1)^{q} \operatorname{det}\left({ }^{\mathrm{t}} g\left(t I_{p, q}-(2 \pi)^{-1} \Omega\right) g\right)=f(t, \Omega) .
\end{aligned}
$$

This completes the proof.

It is to be noted that $f(t, \Omega)$ is an $\operatorname{ad}\left(\mathrm{SO}_{0}(p, q)\right)$-invariant form in the following sense. Put

$$
f^{\prime}\left(t, \Omega^{\prime}\right) \equiv f\left(t, I_{p, q} \Omega^{\prime}\right)=f(t, \Omega) .
$$

Since $\Omega^{\prime}$ is transformed according to $(1)$, we easily see the invariance as follows:

$$
\begin{aligned}
f^{\prime}\left(t, \bar{\Omega}^{\prime}\right) & =f^{\prime}\left(t, g^{-1} \Omega^{\prime} g\right)=f\left(t, I_{p, q} g^{-1} \Omega^{\prime} g\right) \\
& =f\left(t,{ }^{\mathrm{t}} g I_{p, q} \Omega^{\prime} g\right) \quad\left[\text { i.e., } I_{p, q} g^{-1}={ }^{\mathrm{t}} g I_{p, q}\right] \\
& =f\left(t,{ }^{\mathrm{t}} g \Omega g\right)=f(t, \Omega)=f^{\prime}\left(t, \Omega^{\prime}\right) .
\end{aligned}
$$

Associated with $f(t, X)$, we can define $\operatorname{ad}\left(\mathrm{SO}_{0}(p, q)\right)$-invariant polynomial functions $\bar{g}_{0} \cdot \bar{g}_{1} \ldots, \bar{g}_{n}$ on $\mathfrak{s o}(p, q)$ by

$$
f(t, X) \equiv \sum_{i=0}^{n} \bar{g}_{i}(X) t^{n-i} \quad \text { for } X \in \mathfrak{s o}(p, q) .
$$

For a $2 k$ th function $\bar{g}_{2 k}$ there exists a unique closed $4 k$-form $\bar{\beta}_{k}$ on $M$ such that $\pi^{*}\left(\bar{\beta}_{k}\right)=\bar{g}_{2 k}(\Omega)$, where $\pi: \xi \rightarrow M$ is the projection. We denote by $\overline{\mathfrak{p}}_{k}$ the class of the $4 k$ th de Rham cohomology $\operatorname{ring} H^{4 k}(M ; \mathbf{R})$ represented by $\bar{\beta}_{k}$ and call it the $k$ th pseudo-Pontrjagin class of $M$. Put $\overline{\mathfrak{p}}=1+\overline{\mathfrak{p}}_{1}+\overline{\mathfrak{p}}_{2}+\cdots$ and call $\overline{\mathfrak{p}}$ the total pseudo-Pontrjagin class of $M$.

We now state the main theorem. 
MAIN THEOREM. Let $M$ be an n-dimensional orientable pseudo-Riemannian manifold of metric signature $(p, q), p \geq q, p+q=n$. Then the pseudo-Pontrjagin class $\overline{\mathfrak{p}}$ of $M$ coincides with the Pontrjagin class $\mathfrak{p}$ of $M$.

PROOF. In $[\mathbf{1}]$ Chern observed that at each point $x \in M$, the tangent space $\pi^{-1}(x)$ splits into the $p$-dimensional subspace $\pi_{+}^{-1}(x)$ and the $q$-dimensional subspace $\pi_{-}^{-1}(x)$ with respect to the indefinite metric of signature $(p, q)$, together with a Riemannian metric on $M$. Since such a splitting is continuous and global. this implies that $M$ admits a continuous field of tangent $q$-planes (see also [3. $\S 40]$ ). Thus, we can always choose a local pseudo-orthonormal basis $\left\{e^{i}\right\}$ of 1-forms on $M$ so that $e^{1} \ldots \ldots e^{p}$ lie on $\pi_{+}^{-1}(x)$ and $e^{p+1} \ldots . e^{n}$ lie on $\pi_{-}^{-1}(x)$. Relative to this basis the curvature 2 -form $\Omega$ can be written as a diagonal block matrix

$$
\left.\Omega=\left[\begin{array}{cc}
\frac{p}{\Omega_{(+)}} & \frac{q}{0} \\
0 & \Omega_{(-)}
\end{array}\right]\right) \quad \begin{aligned}
& p \\
& q
\end{aligned} .
$$

It is important to note that in this form $\Omega$ is compatible with the intrinsic Riemannian structure on $M[\mathbf{1}]$. Therefore, inserting such an $\Omega$ into $f(1, \Omega)$, we have

$$
\begin{aligned}
f(1, \Omega) & =(-1)^{q} \operatorname{det}\left(I_{p . q}-(2 \pi)^{-1} \Omega\right) \\
& =(-1)^{q} \operatorname{det}\left(I_{p}-(2 \pi)^{-1} \Omega_{(+)}\right) \wedge \operatorname{det}\left(-I_{q}-(2 \pi)^{-1} \Omega_{(-)}\right) \\
& =(-1)^{q} \operatorname{det}\left(I_{p}-(2 \pi)^{-1} \Omega_{(+)}\right) \wedge \operatorname{det}{ }^{t}\left(-I_{q}-(2 \pi)^{-1} \Omega_{(-)}\right) \\
& =(-1)^{q} \operatorname{det}\left(I_{p}-(2 \pi)^{-1} \Omega_{(+)}\right) \wedge \operatorname{det}\left(-I_{q}+(2 \pi)^{-1} \Omega_{(-)}\right) .
\end{aligned}
$$

where the last equality holds since ${ }^{\mathrm{t}} \Omega_{(-)}=-\Omega_{(-)}$.

If $q$ is odd then the second part of the last expression becomes

$$
\operatorname{det}\left(-I_{q}+(2 \pi)^{-1} \Omega_{(-)}\right)=-\operatorname{det}\left(I_{q}-(2 \pi)^{-1} \Omega_{(-)}\right) .
$$

and, hence,

$$
\begin{aligned}
f(1, \Omega) & =(-1)^{q+1} \operatorname{det}\left(I_{p}-(2 \pi)^{-1} \Omega_{(+)}\right) \wedge \operatorname{det}\left(I_{q}-(2 \pi)^{-1} \Omega_{(-)}\right) \\
& =\operatorname{det}\left(I_{p+q}-(2 \pi)^{-1} \Omega\right)=\operatorname{det}\left(I_{n}-(2 \pi)^{-1} \Omega\right) .
\end{aligned}
$$

If $q$ is even

$$
\operatorname{det}\left(-I_{q}+(2 \pi)^{-1} \Omega_{(-)}\right)=\operatorname{det}\left(I_{q}-(2 \pi)^{-1} \Omega_{(-)}\right) .
$$

and, therefore,

$$
\begin{aligned}
f(1, \Omega) & =(-1)^{q} \operatorname{det}\left(I_{p}-(2 \pi)^{-1} \Omega_{(+)}\right) \wedge \operatorname{det}\left(I_{q}-(2 \pi)^{-1} \Omega_{(-)}\right) \\
& =\operatorname{det}\left(I_{p+q}-(2 \pi)^{-1} \Omega\right)=\operatorname{det}\left(I_{n}-(2 \pi)^{-1} \Omega\right) .
\end{aligned}
$$

Thus, irrespective of type of signature, the pseudo-Pontrjagin class $\bar{p}$ represented by a form $\bar{\beta}$, with $\pi^{*}(\bar{\beta})=f(1, \Omega)$, coincides with the Pontrjagin class $\mathfrak{p}$ which is represented by $\beta$, with $\pi^{*}(\beta)=\operatorname{det}\left(I_{n}-(2 \pi)^{-1} \Omega\right)$.

Although we treated the (pseudo-)Pontrjagin class of the tangent bundle of $M$ with an indefinite metric, these results are easily applicable to general vector bundles with indefinite metrics.

I would like to thank Professor Chiaki Ihara for valuable discussions and encouragement. 


\section{REFERENCES}

1. S. S. Chern, Pseudo Riemannian geometry and the Gauss-Bonnet formula, Acad. Brasil. Ciencias 35 (1963), 17-26.

2. Y. Matsushita, Thorpe-Hitchin inequality for compact Einstein 4-manifolds of metric signature $(++$ - -) and the generalized Hirzebruch index formula, J. Math. Phys. 24 (1983), 3640.

3. N. Steenrod, The topology of fibre bundles, Princeton Univ. Press, Princeton, N. J., 1951.

Department of Applied Mathematics and Physics, Faculty of Engineering, KYOTO UNIVERSITY, KYOTO, JAPAN 